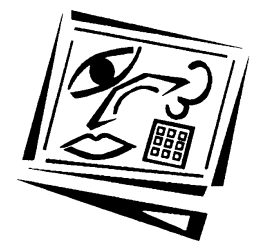

\title{
Students' perception of supplementary, online activities for Japanese language learning: Groupwork, quiz and discussion tools
}

Keri Moore and Sumiko Iida

University of New South Wales

\begin{abstract}
This paper discusses the frequency of use and the reasons for non-use of the online component of a course developed in Blackboard 9, one popular learning management system (LMS) in tertiary education. The study particularly focuses on the three tools in the LMS: Groupwork tool, Downloadable quiz and Discussion forum. These tools were used in a Japanese upper beginners-level course in 2009. At the end of the semester, a student survey was conducted. The survey result indicates that although use of the online component of the course and of the individual tools differed, the frequency of the student use of online supplementary course materials and tools has an effect on their positive perception of the educational value, the development of scholarly skills, that this type of teaching strategy makes. The frequency of student use is determined by their attitude towards online learning, their ability to use the different functions, their peer's use of the same tools, and their access to computers.
\end{abstract}

\section{Introduction}

Among recent developments in educational technology, learning management systems such as WebCT Vista, Blackboard and Moodle, have become widely used to supplement or enrich courses and to provide learners with an alternative learning environment. Embedded in the notion of introducing more technology in higher education is the assumption that current university students have computer and broadband access and that they can use, or can learn to use the technology effectively. This is a chicken and egg issue: the students' ability to use the technology enables them to participate in tertiary education and developments in technology results in the need for educators and students to learn more ways to engage in tertiary education.

The current trend in the higher education sector is to progressively introduce more online course modules to courses within all manner of programs. Whether students fully utilise online components of courses and individual tools, and whether they view these strategies as adding value to their educational experience is not known. Because students use one kind of technology in their personal lives it cannot be assumed automatically that they will be immediately competent in the effective use educational technology. If students are not comfortable with and have no interest in using technology, then any intended educational value will not be realised. Thus it should not be automatically assumed that students like to learn through technology.

The course used for this study was an upper beginners level of Japanese communication course, taught in 2009 by one of the authors of this paper. Since the introduction of LMS at the University of New South Wales, this course and its 
prerequisite courses were taught using WebCT Vista. Prior to the whole university shifting its use of LMS product to Blackboard 9 from 2010, the course tried out Blackboard 9 as a pilot with 17 other courses across the university. Using Blackboard 9 was therefore the first time for both the instructors and the students. In this iteration the following tools were used:

a. The Groupwork tool

b. The Online downloadable quiz

c. The Discussion tool

The evaluation research project, which was non-experimental, explored the relationship between the frequency with which students used the online course and the different tools and their perception of educational value of the same. It is not expected that a clear cause and effect relationship will be demonstrated; rather we aim to identify the students' voice on the use of technology in this course.

The questions were:

1. What is the students' frequency of use, and what are the reasons for the non-use of the online coursework and tools, and how do they use or do not use them?

2. What is the students' perception of the educational value of the tools and is that associated with their frequency of use of the tools?

What constitutes an educationally effective LMS is extracted from the extensive literature on higher education e-learning, and e-teaching. For the purposes of this study the key elements of a valuable supplementary online course were the development of a scholarly approach to learning, engagement in a scholarly community, and the online component facilitating the students' progression in the course.

By doing so, the aim of the study is to expand on the current scholarly discourse concerning the use of technology in tertiary language education. Previous research on the use of technology in second/foreign language teaching focused on: the effectiveness of the computer-mediated communication as a learning and teaching resource (Ramzan \& Saito, 1998); the use of a telecollaborative learning environment (Belz \& Kinginger, 2002); audiographic conferencing (Hampel, 2003); electronic discussion (Hanna \& de Nooy, 2003); synchronous chat systems used to explain technical concepts to student (Day, Wood, Scutter \& Astachnowicz, 2003); online role plays ( Milton, 2004); students use of electronic course supplements, such as film to improve their levels of proficiency (Bueno, 2006); the development of a tool to evaluate oral tests (Laborda, 2006); the use of computer technology in the assessment (Hunt, Neill \& Barnes, 2007); problems and strategies in oral communication tasks (Lam, 2007); online interaction with native speakers (Lee, 2004; Mahfouz \& Ihmeideh, 2009); Learners' scaffolding using social network service (Christensen, 2010); and video presentation on students' blogs to enhance public speaking skills (Shih, 2010).

It was determined from the above review that there is a scarcity of information about the students' frequency of use of online tools and the reasons for their use or non use in a second/foreign language learning situation. Further, there is limited literature focusing on the students' perception of the educational value of online supplementary course work and tools. 


\section{The structure of the course studied}

The key objectives of the target course are to develop effective communication skills, knowledge skills and ability in Japanese. In this course, the learning activities included lectures, tutorials and online activities. The general aims of the online component of the course were to:

- encourage students to become an autonomous learners, by exchanging resources and ideas with fellow students in discussion and group work;

- follow up the class content of the week using discussion, homework and quiz; and

- provide students with opportunities to monitor and assess their own writing and oral presentation skills in comparison with others, for further improvement of presentation skills through group work.

The majority of students in the course were continuing students of the Japanese Communication course offered in the previous semester, where students participated in a few online tasks including video and voice presentations on WebCT Vista. This time, amongst a variety of tools on Blackboard 9, students were encouraged to use three online tools, mentioned above. The development of these tools was underpinned by two key educational theories. One is adult learning theory (Knowles, Elwood \& Swanson, 1978). This theory posits that adults bring experience to the learning setting, which educators can use as a learning resource for all students. Further, adult learners are known to prefer to provide input into how the learning activities are structured, and they learn better when they see the relationship between the activity and the desired learning outcome. The other theory is social learning theory (Bandura, 1986), which posits that people learn from each other via observation, imitation and modelling. That is, by the very act of socialising in a culture people learn the customs, social and political practices, morals and ethics of a particular culture.

With these theories in mind, the Groupwork tool was chosen so that students could develop one of their assignments: public speech in Japanese. It was envisaged that through the use of the Groupwork tool, students would draft the videos presentation of their speeches and share them with classmates so that they could receive and give feedback. Using the Groupwork Tool was assumed to develop the students' understanding of Japanese, ability to share and to work in a group, to communicate, their ability to reflect, critically evaluate, and to study independently.

In contrast, the Online downloadable quiz was developed with the notion that students wishing to achieve their greatest potential in the course would engage in practice exercises to develop their knowledge and skills. The quiz was not an assessable task but for the revision of the course content. The quiz took a variety of formats such as ordering phrases, gap-fill, match-up, and rewriting sentences. The aim of using this tool was to develop the students' understanding of Japanese, skills in reflection, and capacity for independent study.

Using a Course discussion tool was expected to enable students to continue the topics of discussion they held from classes in every week. Students were also encouraged to use the tool for community collaborations, to exchange information and ideas about Japanese culture. It was envisaged that by using this tool, students would enhance one another's understanding of Japanese, sharing of knowledge, communication, and skills in reflection and in using information technology. 
Due to the nature of the course, different types of assessment tasks including quizzes, tests, presentations, homework and an exam, were set to assess student's reading, writing, listening and speaking skills. In addition, the students were advised that they were required to access Blackboard 9 at least twice a week throughout the semester. The expectation was that by regular access to the supplementary course materials and learning strategies, students would develop the necessary skills to be successful in the course. It was also hoped that in doing so, students would experience a stronger sense of community and a friendlier, more productive learning environment.

The aims of this evaluation then, was to contribute to the literature in e-teaching and elearning by exploring the success of our endeavours, as far as the students perceived them to be.

\section{Methodology}

Consistent with the approach to evaluation research outlined by Charles and Mertler (2002), the data sources included 121 students enrolled in the 2009 offering of the course, who all used the Blackboard 9 and the three tools: Groupwork tool, Quiz tool and Discussion tool.

At the beginning of the semester, students were informed about the study and introduced to the use of Blackboard 9, particularly these tools during the course.

For the group work, students were firstly asked to sign up to a group, then to select applications (available under the Group work tool) they wished to use prior to the actual group activity. Once they settled in each group, they were advised to commence their work by 1) video record their self introduction in Japanese, and embed the video via YouTube to get to know each other, 2) exchange the idea of the speech topic, 3) upload speech drafts (up to the second draft uploading was mandatory), 4) video record the presentation practice. All of these semester-long tasks encouraged students to comment upon posts by others in the group.

For the quiz, out of 5 chapters taught during the semester, 3 were made available online, including PDF files of similar quizzes for the students to download. Quizzes for the last two chapters were not made online due to a variety of formats that were difficult to fit online. Each quiz was released in the week the chapter was completed for students to self-test their knowledge and skills.

As for the discussion tool, three forums were created: weekly course related discussion, Japanese popular culture discussion and Japanese traditional culture discussion. These forums were open to students to share their questions, answers, opinions, requests and experience of the course and Japanese culture.

Of these three tools, the discussion tool did not require any instruction as it had been commonly used often by students in their earlier courses. Also, instruction for the quiz tool was not necessary, but students were advised every time a new quiz was released. However, the group tool needed a clear technical instruction, particularly on video embedding. Thus, an information sheet was created and uploaded to the Blackboard 9 site, and a demonstration of the group work was given in the lecture. Students who experienced technical problems during the semester contacted the instructor for individual support. 
Data was collected by a survey presented as hard copy and conducted in the last class of the semester. Students completed the survey anonymously.

The validity of the survey content and its construction were strengthened by the preparation process which included the extensive literature search and also a review by several groups of academics. The majority of questions/statements were quantitative in that they required students to respond on a Likert scale, either: 'Strongly agree'; 'Agree'; 'Neither agree nor disagree': 'Disagree' or 'Strongly disagree'. In addition, there were some qualitative, open ended questions in which students were asked to give examples or further explain their responses to the quantitative questions. Plus, the students were asked to indicate if they accessed the tool frequently, moderately, less than expected or hardly ever. Where applicable, the students were asked to select from a number of choices, the reasons for their non-use of the course materials.

All data was entered into SPSS and in the first stage of analysis the students' responses were analysed for frequency. During that phase of data analysis, deductive analysis showed that even with visual examination, patterns of responses were clearly different based on student's answers to the question about how often they used a tool. Therefore, the responses to questions about the three tools were divided into two groups for analysis.

- Group 1 included the responses from those students who had accessed the online tool moderately or frequently.

- Group 2 included the responses from those who had accessed it either less than expected or hardly ever.

The following tests were then applied. Firstly, a Multivariate analysis (MANOVA) technique was used to determine the effects of independent categorical variables on multiple continuous dependent variables. This test is usually used to compare several groups with respect to multiple continuous variables. The dependent variables included three tools. Because we asked how often students accessed the tools and about their perception of its educational value according to the intended use of the tool, it meant that eight quantitative questions were asked about the Groupwork tool; three about the Downloadable Quiz and six about the Discussion tool.

Secondly, a Hotelling trace coefficient was utilised, which presents a statistic for a multivariate test of mean differences between two groups. Thirdly, Levene's test of equality of error variance was used. It tests the null hypothesis that the variances of the group are the same. If this is greater than a selected percentage (usually 5\%) then it is considered too great to usefully apply parametric tests.

The qualitative comments underwent inductive analysis in that they were analysed manually which allowed categories of themes to be developed (Charles \& Mertler, 2002). The results are presented below.

\section{Results from the survey}

One hundred and eleven (111) of the 121 enrolled students responded to the survey, giving a high response rate of $91.7 \%$. 


\section{Analysis phase one: Students' views of the online resources in general}

For data presentation, the 'Strongly agree' and 'Agree' responses were combined, and likewise 'Disagree' and 'Strongly disagree' were combined. Of the 111 respondents, 86 agreed that omnline resources enhanced their learning experience. The particular views of the educational contribution they believe the online course supplements made to their learning are presented below, with numbers given for the combined 'Agree' responses.

The online resources helped the development of scholarly skills such as the:

- preparation for class $(\mathrm{n}=70)$

- preparation for assessment $(\mathrm{n}=66)$

- integration of course work $(n=74)$

- exploration of the subject matter more deeply $(n=42)$ $(n=46$ neither agreed nor disagreed).

The online resources helped students develop a sense of a scholarly community ( $n=52)$. In particular the:

- ability to express and share ideas $(n=60)$

- ability to interact with teachers and tutors $(\mathrm{n}=54)$

- ability to seek and receive feedback from teachers $(\mathrm{n}=72)$

- ability to seek and receive feedback from other students $(\mathrm{n}=61)$

- added great value to the students' face to face interactions $(n=57)$.

The online resources assisted the students' progression in the course $(n=79)$. They also said that:

- having the flexibility to study at suitable times suited me ( $\mathrm{n}=84)$; and

- having that option had a significant impact on the students' ability to complete the course $(n=64)$.

The qualitative data shows a trend for positive comments about:

- Communication: "The discussion system in Blackboard 9 really helps in doing group activities and it also provides a good platform for collaborative work between group members".

- Resources: "Make course material accessible".

- Functionality: "Submitting work was easier." A few other comments were negative or non committal or that they preferred to use WebCT Vista.

The students' responses to questions about how often they accessed the online Blackboard 9 module in general were as follows:

- the majority, accessed it a few times a week as required in the course $(\mathrm{n}=64)$

- less than once a week $(\mathrm{n}=28)$

- hardly ever used it $(\mathrm{n}=9)$

- used it more than a few times per week $(\mathrm{n}=10)$.

About one half of the students $(n=57)$ gave reasons for their non-use of the online supplementary course activities. Specifically they reported: 
- do not like Blackboard 9 in particular $(n=31)$;

- do not like online modules in general $(n=13)$; or

- the home environment was not amenable $(n=7)$; or

- that they did not like to learn through technology $(n=2)$.

\section{Students' thoughts on the Groupwork tool}

The students' report on their use of the Groupwork tool (Table 1) was follows:

- Frequently $(\mathrm{n}=2)$

- Moderately $(n=38)$

- Less than expected $(\mathrm{n}=50)$

- Hardly ever $(\mathrm{n}=12)$

Table 1: The students' responses about the three tools

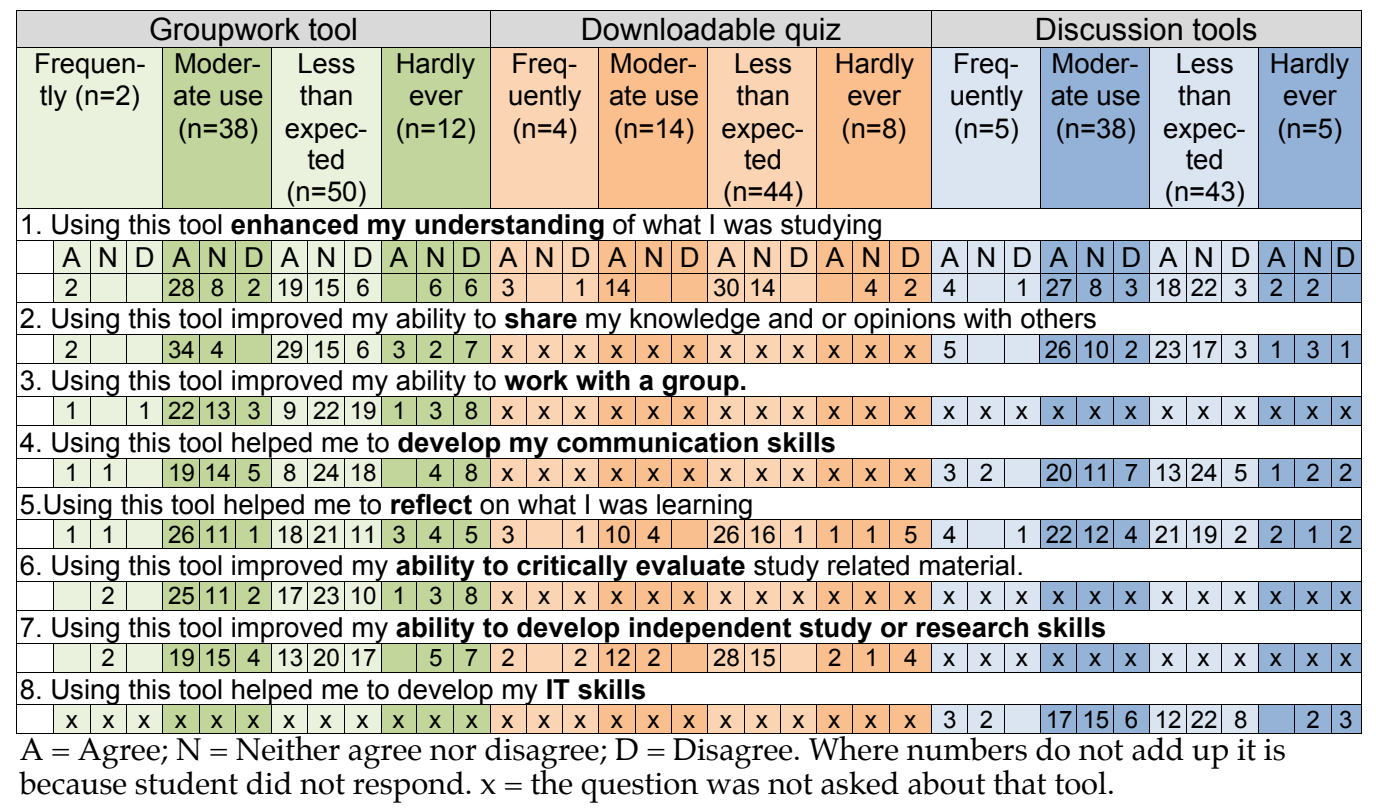

Hotelling's Trace found $F(16,184)=2.616$ and $(p<0.001)$. This means that students perceived the Groupwork tool differently depending on their frequency of use, their level of involvement overall. There was one exception: the analysis of the question regarding the statement the Groupwork tool improved my ability to share my knowledge and opinion with others', showed that the error variance was not equal according to Levene's Test, which turned out to be significant. So the results of the students' response to that statement will be interpreted with caution. Table 1, shows the spread of students' responses to questions about the Groupwork tool.

The qualitative data showed the following themes emerged from the students' comments about the lack of use of the Groupwork Tool. They were:

- The inactivity of other students: "No one reviewed my drafts so I didn't bother to review others." 
- It was difficult to use: "I found that these things required so much effort. More effort than usual. I see how it could work and could be of benefit but other students hardly used it so I found that I was talking to myself most of the time, then I completely neglected it. I do however, like the idea of group work, just not when it is a hassle logging in, posting, etc."

- A lack of preference for online discussions: "Particularly in a language course, I believe group work should be face to face if it is to be constructive to our learning."

When asked, "what was the best thing about using the Groupwork tool?", the general consensus was captured by the following comments, which balance the negative comments above. The best things were:

- "Interaction with others";

- "Being able to view other students' work to get a better understanding of the work";

- "Sharing information"; and

- That "someone else was able to give me suggestions without the awkwardness that I might have when asking teacher questions."

The suggestion for improvement included a call for more instruction of using the tool. Given the assumption that the Groupwork tool would develop students' skills such as a better understanding of Japanese, ability to share and to work in a group, to communicate, their ability to reflect, critically evaluate and study independently, the aim was realised overall. The exception was that it seems a number of students did not share their work with others.

\section{The student thoughts on the Online and Downloadable Quiz}

The students report on the frequency of their use of the Quiz (Table 1) was as follows:

- Frequently $(\mathrm{n}=4)$

- Moderately $(\mathrm{n}=14)$

- Less than expected $(\mathrm{n}=44)$

- Hardly ever $(\mathrm{n}=8)$

Hotelling's Trace found $F(3,66)=2.856$ and $(\mathrm{p}<0.05)$. Here again this means that the students rated the tool differently depending on their frequency of use of it for the most part. The exception was the analysis of the question that asked if the Quiz enabled students to better understand the course material showed that Levene's Test was significant so, the results of that question should be interpreted with caution. Table 1 shows the spread of the students' responses to questions about the Quiz. The qualitative data confirms the above quantitative data in that it showed that students thought the best thing about using the downloadable quiz was that it assisted their learning. Students said the online quiz gave them the ability to learn more and to revise and to prepare for exams/quizzes. It also allowed them independence in their pace of study and the flexibility to study at times that suited them.

Suggestions for improvements included requests for more flexibility and more information and to be able to retake the test; to allow the tests to be retaken several times and for answers to be provided. Also, some students wanted more precise rewards for effort; they wanted the results of the quiz to be included in their grades. 
The aim of selecting the quiz tool was to develop the students' skills understanding of Japanese, reflection and independent study, and this seems to be true with the exception that improving their understanding was not as clearly established.

\section{Student thoughts about the Discussion tool}

The students reported use of the Discussion tool (Table 1) was as follows:

- Frequently $(\mathrm{n}=5)$

- Moderately $(\mathrm{n}=38)$

- Less than expected $(n=43)$

- Hardly ever $(\mathrm{n}=5)$

Hotelling's Trace found $F(5,87)=2.433$ and $(p<0.05)$, this means that the difference in responses related to frequency of use was smaller than in relation to the other two tools, but it is still significant. Levene's Test showed that the students' responses to the question about the Discussion tool helping them to improve their communication skills should be interpreted with caution. Table 1, shows the spread of the students responses to questions about the Discussion tool.

With regard to the qualitative data, only a handful of students gave reasons why they did not access the discussion tool. They were as follows:

- "I felt that I had nothing to contribute"

- "I find it's not a very useful way to get answers"

- "I didn't really need to"

When asked, "what was the best thing about using course Discussion tool?", the general sentiment was that the tool allowed them to: ask the questions immediately and get a response as soon as possible; and to share with everyone in the course, rather than only the people in the group. Suggestions for improvement were: Topic search and sorting.

It was anticipated that the discussion tool would enhance student mutually understanding of Japanese, sharing of knowledge, communication, skills in reflection, and in using information technology, all seemed to be realised except there are some questions over the development of communication skills that have not been resolved.

\section{Discussion}

Following a summary of the survey results above, this section discusses some circumstances that may have impacted on the results. This section also discusses the implications of the current students' attitudes towards online course modules, and suggests how to proceed in the future.

The qualitative comments suggest that some students seemed to struggle to adjust themselves to a new LMS environment. This may be due to the fact that the use of Blackboard 9 in the course was limited to only 18 courses amongst over a few thousands of courses where WebCT Vista was mainly used. As a result, the students had to use two different LMS applications for the courses they studied in the semester. 
Even so, the majority of the students accessed Blackboard 9 a few times a week as required in the course. Well over half the respondents thought that it helped them to develop scholarly skills such as preparation for class and assessment, and that it integrated their course work, that it helped them feel part of part of what? and to develop a scholarly community, to express their ideas, interact and seek and receive feedback, and it added value to their face to face interactions. The students also said the LMS had a positive impact on their progress in the course. The only area in which the students had mixed views was in relation to whether or not the online module helped them explore the subject matter more deeply. This means that the students' low access to the course module does not directly infer that they do not value online learning as an effective education medium.

Closer inspection of the $(n=57)$ students' responses outlining reasons for their non-use of the online supplementary course activities found that they did not like Blackboard 9 in particular, $(n=31)$, they do not like online modules in general $(n=13)$, and the home environment was not amenable $(n=7)$. Only two students entered their negative views on learning with technology. These numbers are significant and cannot be ignored. For the students who are not keen on technology, a hands on exercise is needed. Also, it would be advantageous if such students learned that the online course modules are not as difficult as they believe at the beginning of the semester. For students who do not have broadband access at home, it is a difficulty, but this is a reality and we should not expect that it is always the case that students can afford broadband access. Of course, the university has a number of computers for students to use on campus, and perhaps such students can use them to do the tasks or submit assignments, but students recount that these computers are not convenient for updating course related information at the pace that is required by the instructor. This home computer network problem seems to be always the case for some students (e.g. Shih, 2010). In order for students to catch up with the course related information it may be useful to trial a link between the course module and their mobile phones or portable electronic device, which almost everyone uses nowadays.

The following are reflections upon the results with each of the three tools used in the course.

\section{The Groupwork tool}

The utilisation of Groupwork was the first attempt for this course since it started in 2001. The data show that the majority of students used this tool less than expected, and in addition to the student comments about the lack of use by their peers, there are other reasons to consider. A number of problems were experienced, and they seem to be reflected in the student qualitative responses. First, all group applications were made available to students to choose what they believe to be effective for their work. However, students seemed to have become confused or unsure of how each application would work, which caused a very slow start of the group work for the majority of the students. Thus, a recommendation of blog and discussion use was given to all students later.

Secondly, a major technical problem was encountered in the uploading of the selfintroduction videos students had recorded using a webcam. It was one of the required activities in the group work for the purpose of getting know each other quickly. During the Blackboard 9 training session for teachers, it was advised that the video 
upload could be easily done by copying and pasting embedded code in the HTML window of the text editor. However, it was not until the students started to work on the task that we realised that the function was available only on the teacher window with the 'editor' button, despite the text editor in the student window showing a tool icon to embed video streaming. Students who followed the video embedding process thus ended up failing. After a number of consultations and discussions, a way to upload a video from the student window was found, but it required a complex editing of the original embedded code. As a result, many students could not handle the technology and the instructor had to intervene. Students seemed to have been discouraged from uploading videos since then and no student posted a video of presentation practice.

Thirdly, as one student commented, there were students who attempted to use the function actively, but they were discouraged by the inactivity of other students who did not post anything, apart from a required initial video introduction, and thus gave up the group communication on the tool.

Fourthly, although the public presentation itself is an assessable task, no clear mark was allocated to its preparation using this group work tool. Thus, it seems that many students ignored it.

These points suggest that the Groupwork tool did not work as effectively as had been expected. Which one of these factors most directly affected students' inactive attitude in the use of the Groupwork tool is unknown. In fact, many students wrote positive comments about the tool. Given these responses it is assumed that solving of the technical problems would enhance the students' use of the tool in future classes. In order to achieve this goal, it would be ideal for students to give feedback to each other on their work in progress. It would also be useful if they could rehearse their own presentations in terms of verbal and nonverbal style, prior to the actual presentation.

\section{Online and Downloadable Quiz}

The online quiz tool was introduced in the course for the first time because it was expected to help students to revise the course contents. In earlier iterations of the course, the quizzes were used as class tests, but because of the shorter semester, which reduced face to face class hours, this activity was pushed online and was used as a non-assessable task. Unfortunately, it is clear that the number of students who used this tool was small $(n=43)$, which was a much less access than had been expected. Even so, students' comments were positive about the tool's contribution to their independent study and ability to revise.

A few students pointed out the technical inflexibility of the online quiz. It was indeed difficult to create a quiz for a character based language course. The match-up, and multiple answer type of questions were not the problem, but when making quizzes that ask students to write in such as a gap filling quiz, some difficulties were encountered. The computer simply marked as wrong some answers that should have been marked as correct had they been completed on paper and marked manually. Thus, unfortunately, we must admit that this tool (particularly online quizzes) or the way it was used in the course was premature, and did not work effectively as had been expected. Finally, a few students suggested including the quizzes as a part of an assessment task. The reason why it was not included in the formal grade was due to 
concerns regarding academic misconduct. This is a task that can be carried out by anyone, which means if students wish to strive for a higher mark, they might easily ask a native speaker of Japanese or a senior student of Japanese to do it.

\section{Discussion tool}

The discussion tool is one of the most popular tools used in the LMS. It allows the teacher to post announcements and students to post course related questions to which peers and the teacher can reply. It also provides the users with a space to exchange ideas and opinions about a certain topic. Compared with the other tools, students were most familiar with this tool in the previous LMS and hardly ever had any technical problems in using it in Blackboard 9.

Anecdotal evidence suggests that both teachers and students felt uneasy using this tool compared with the one on WebCT Vista. Unlike WebCT Vista in which only one discussion can have multiple categories where multiple topics can be set and viewed without leaving the top page, Blackboard 9 allows only access to one forum at a time. Thus for example, after reading a post under a weekly course related discussion forum, in order to see a post in Japanese culture discussion, the student must go back to the top page. Students who had been used to discussions on WebCT Vista became very frustrated by this, and as a result, the number of total posts in the discussion tool on Blackboard 9 was only 168, which was a large decrease compared to 398 in the previous semester when WebCT Vista was used.

The results show that the majority of students used the Discussion tool less than expected $(n=43)$, yet the majority agreed the tool helped them to share information, improved understanding and reflection, but not apparently, their communication skills. Although most of the students' comments were what one would typically expect, such as they felt they had nothing to contribute, others liked the idea of instant discussions. Three students made particular comments that they preferred WebCT Vista for this particular tool.

\section{Conclusion}

The findings depict the current student attitudes towards online course activities. The frequency pattern of students' use of these tools reflects what the course requires of the student rather than what the course offers them. From this, it can be argued that current students are not very active users of LMS, and use it only for the activities directly related to the course assessment.

When the findings are considered against the principles of adult learning theory (Knowles, Elwood \& Swanson, 1978) it seems that students may bring their preconceived ideas about learning via technology and it may not be possible in every instance to overcome their preconceptions. As far as social learning theory (Bandura, 1986) is concerned, the lack of use of the tools in this iteration of the course limited the possibility for students to learn from each other.

It is clear from this study that the frequency with which students use online supplementary course materials has an effect on their positive perception of the educational value of the tools. However their frequency of use is determined by course requirements, student attitudes to online learning, their ability to use the different 
functions, their classmate's use of the same, and their access to computers. What cannot be determined is, whether at the outset students held preconceptions about online learning activities being a negative experience. Alternatively, they may have had a positive expectation and tried the online activities in this course and did not like them, or they may not have wanted to try.

To answer our first question: "what is the students' frequency of use, and what are the reasons for the non-use of the online coursework and tools and how they use or do not use them?": of the 57 students $(52 \%)$ who gave reasons for their non-use of the online supplementary course materials, $(\mathrm{n}=31,29 \%)$ were unsatisfied with Blackboard 9 itself and $(\mathrm{n}=13,12 \%)$ do not like online learning. Given this result, the main reason for student non use of the online course module is due more to the application itself rather than LMS per se. However, it is necessary to point out that the majority of the students had been using WebCT Vista for more than a year since they entered the university and were used to using it. Thus, it seems that the problem they encountered more in the transition to a totally new application with a completely different structure than in using the Blackboard 9 itself.

To answer our second question: "what is the students' perception of the educational value of the tools and is that associated with their frequency of use of the tools?": the answer seems to be 'yes', there seems to be a significant difference in perception of usefulness of the tools depending on the frequency of use.

\section{Future research}

In the next iteration of the course a comparative study using Moodle is under consideration. Though this course did not mention it, Blackboard 9 has a function to synchronise iPhone and Facebook. This can be fully utilised to encourage students to use the learning module more effectively. We did not investigate the students' use of social network services (SNS) this time, but if more students use SNS than the online course module, there is no reason to use SNS as a mediating application to the LMS.

\section{Acknowledgments}

The authors wish to acknowledge the funding and support of the Faculty of Excellence Initiative at The University of New South Wales and members of the staff who participated in the initiative. We would also like to acknowledge the considerable contribution of Lynn McDonald (http://www.infomanagerplus.com.au/) and thank her for her expertise and professionalism. We also thank Mariya Pachman from L\&T $@$ UNSW for her statistical analysis and advice. The study received HREA Ethics Approval Number 092072.

\section{References}

Abrams, Z. I. (2003). The effect of synchronous and asynchronous CMC on oral performance in German. The Modern Language Journal, 87(2), 157-167.

Bandura, A. (1986). Social foundations of thought and action: A social cognitive theory. Englewood Cliffs, NJ: Prentice-Hall.

Belz, J. \& Kinginger, C. (2002). The cross-linguistic development of address form use in telecollaborative language learning: Two case studies. The Canadian Modern language Review, $59(2), 189-214$. 
Bueno, K. (2006). Stepping out of the comfort zone: Profiles of third-year Spanish students' attempts to develop their speaking skills. Foreign Language Annals, 39(3), 451.

Charles, C. \& Mertler, C. (2002). Introduction to educational research (4th ed.). Boston, MA: Allyn and Bacon.

Christensen, M. (2010). Impediments to linguistics scaffolding in computer mediated language learning. Paper presented at the 35th Applied Linguistics Association of Australia, University of Queensland, Brisbane, 4-7 July 2010.

Day, I., Wood, D., Scutter, S. \& Astachnowicz, S. (2003). Interdisciplinary trials of synchronous, voice-based communication systems. In Interact: Integrate: Impact. Proceedings ASCILITE Adelaide 2003. http: / / www.ascilite.org.au/ conferences/ adelaide03/ docs/ pdf/152.pdf

Hampel, R. (2003). Theoretical perspectives and new practices in audio-graphic conferencing for language learning. ReCALL, 15(1), 21-36.

Hanna, B. E. \& de Nooy, J. (2003). A funny thing happened on the way to the forum: Electronic discussion and foreign language learning. Language, Learning and Technology, 7(1), 71-85. http: / /lt.msu.edu/vol7num1/ hanna/default.html

Hunt, M., Neill, S. \& Barnes, A. (2007). The use of ICT in the assessment of modern languages: The English context and European viewpoints. Educational Review, 59(2), 95-213.

Knowles, M., Elwood, F. \& Swanson, R. (1978). The adult learner: The definitive classic adult education and human resource development. (6th ed.). London: Elsevier.

Laborda, J. (2006). Designing an Internet based tool for oral evaluation in the University Access Examination in Spain. In CALL and monitoring the learner. Proceedings of the International CALL conference, Antwerp, Universiteit Antwerpen, 20-22 August 2006. http:/ / www.eric.ed.gov:80/ERICWebPortal/ contentdelivery/ servlet/ERICServlet?accno=ED504819

Lam, W. (2007). Tapping ESL learners' problems and strategies in oral communication tasks: Insight from stimulated recall. Prospect, 22(1), 56-70.

Lee, L. (2004). Learners' perspectives on networked collaborative interaction with native speakers of Spanish in the US. Language, Learning and Technology, 8(1), 83-100. http: / /lt.msu.edu/vol8num1/ lee/ default.html

Mahfouz, S. \& Ihmeideh, F. (2009). Attitudes of Jordanian university students towards using online chat discourse with native speakers of English for improving their language proficiency. Computer Assisted Language Learning, 22(3), 207-227.

Milton, J. C. P. (2004). From parrots to puppet masters: Fostering creative and authentic language. In B. Holmberg, M. Shelley \& C. White (Eds.), Distance education and languages: Evolution and change (pp. 242-257). [verified 15 Dec 2010] http:/ / hdl.handle.net/1783.1/2363

Ramzan, Y. \& Saito, R. (1998). Computer-mediated communication in foreign language learning: A case of the students of Japanese. In Flexibility the next wave? Proceedings ASCILITE Wollongong 1998. http: / / www.ascilite.org.au/conferences/ wollongong98/asc98pdf/ ramzansaito-0066.pdf

Shih, R. (2010). Blended learning using video-based blogs: Public speaking for English as a second language students. Australasian Journal of Educational Technology, 26(6), 883-897. http: / / www.ascilite.org.au/ajet/ajet26/shih.html

Keri Moore, Learning and Teaching @ University of New South Wales UNSW Sydney NSW 2052. Email: keri.moore@unsw.edu.au

Dr Sumiko Iida, Lecturer and Japanese Studies Program Coordinator School of Languages and Linguistics, University of New South Wales UNSW Sydney NSW 2052. Email: s.iida@unsw.edu.au 